\title{
Quantitative Analysis of Concentration Distribution in Non- Evaporative Diesel Spray with Laser Induced Fluorescence Method
}

\author{
Dai Matsuda1, Kosuke Nagamura', Eriko Matsumura², Jiro Senda ${ }^{2}$ \\ ${ }^{1}$ Department of Mechanical Engineering, Doshisha University, Kyoto, Japan \\ 2 Department of Science and Mechanical Engineering, Doshisha University, Kyoto, Japan \\ ${ }^{*}$ Corresponding author email : cyjg1502@mail4.doshisha.ac.jp
}

\begin{abstract}
In this paper, a quantitative analysis of concentration distribution in non-evaporative diesel spray with Laser Induced Fluorescence method (LIF) is reported. It is well known that changes in fuel injection and ambient conditions have a significant effect on spray characteristics. Although many studies have been conducted on the characteristics of diesel sprays, little is known about the effect of fuel injection and ambient conditions on the dispersion of liquid phase concentration. The purpose of this study is to evaluate the effects of injection and ambient conditions on mixture formation through quantitative analysis of liquid phase concentration distribution in non-evaporating spray. The cross-sectional mass flow of the fuel was obtained using the momentum theory from the measured concentration distribution of the liquid phase using the laser-induced fluorescence method. The relationship between the fluorescence intensity and the liquid phase concentration was estimated using these data. As a result, quantitative spatial distribution of the liquid phase concentration was obtained, and the effects of injection and ambient conditions on the dispersion of the liquid phase concentration were evaluated.
\end{abstract}

Keywords: Concentration distribution, Laser induced fluorescence, Mixing

\section{Introduction}

In diesel engines, the breakup and dispersion processes caused by fuel injection are important factors in mixture formation and have a significant effect on the combustion characteristics. Therefore, it is very important to understand the effects of fuel injection conditions and ambient conditions on the air-fuel mixture formation process in order to improve thermal efficiency and reduce harmful emissions through the improvement of combustion characteristics. In order to simultaneously improve the thermal efficiency and reduce harmful emissions of diesel engines, it is necessary to achieve rapid combustion during the late stage of combustion by activating diffusion combustion. To achieve rapid combustion, high-pressure fuel injection and optimization of the number of nozzle holes are used to accelerate the mixing of fuel, air and combustion gas [1]. It is known that increasing the fuel injection pressure and reducing the diameter of the nozzle holes promotes atomization and evaporation of the fuel spray and dilutes the mixture [2][3]. However, due to the difficulty of quantitatively measuring the spatial distribution of liquid phase concentration, detailed knowledge of the effects of fuel injection pressure and ambient density on the mixture formation process has not been obtained. Laserinduced fluorescence was used to obtain information on the liquid-phase concentration distribution in diesel sprays, and the relationship between fluorescence intensity and liquidphase concentration was estimated. These results were applied to diesel sprays under different fuel injection pressures and ambient density to obtain quantitative spatial distribution of liquid phase concentration. The effects of fuel injection pressure and ambient density on the dispersion of the concentration distribution were investigated. 


\section{Experimental Conditions and Optical System}

In this study, laser-induced fluorescence (LIF) method was used, in which a laser sheet was incident on a cross section of a fuel spray containing a fluorescent tracer mixed, and fluorescence from the fluorescent tracer was captured from a direction perpendicular to the incident light. The test fuel was mixed with 93 vol\% $n$-tridecane and 7 vol\% tetralin as the fluorescent tracer [4]. The fluorescence characteristics of the fuel mixed with $n$-tridecane and tetralin were measured using a spectrometer (Hamamatsu Photonics: C8808 01 PMA 11) at an incident wavelength of $266 \mathrm{~nm}$, and the transmission characteristics of the bandpass filter used for fluorescence spectroscopy are shown in Figure 1. Figure 2 shows the optical system used in the spray measurement experiment. The light source was Nd:YAG laser (Quantel Qsmart850 $\lambda=266 \mathrm{~nm}$ ). The beam passes through a pinhole, is reflected by a dichroic mirror, and is irradiated as a sheet by two cylindrical lenses. A band-pass filter, an image intensifier (Hamamatsu Photonics: C4078-01), and a CCD camera (Hamamatsu Photonics: ORCA-R2) were installed at orthogonal positions. The energy transmitted through the dichroic mirror was measured to correct for the variation in pulse energy. The laser sheet light was assumed to have a Gaussian intensity distribution, and the captured images were corrected. The experimental conditions are shown in Table 1. The fuel injection nozzle was a single-hole nozzle with the injection direction straight down. The injection period was set to be sufficiently long, and the shooting timing was set to $2.0 \mathrm{~ms}$ from the start of injection so that the injection rate and the spray in the visible region would be in a quasi-steady state. The fuel injection

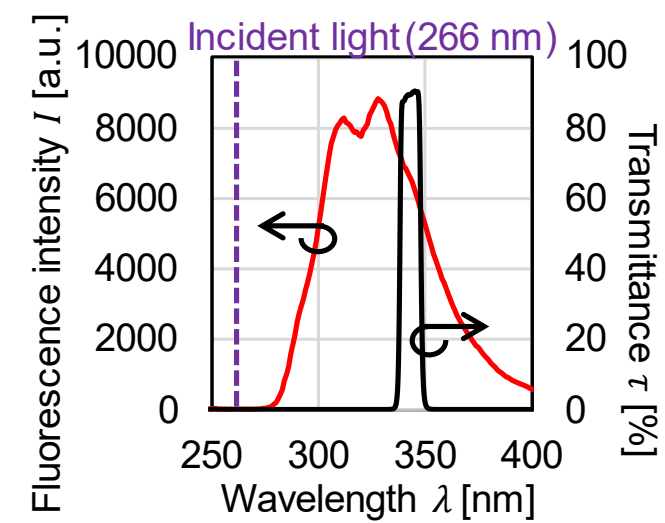

Figure 1. Fluorescence spectra of $\mathrm{nC} 13$ and Tetralin (93:7 vol\%) under 266nm excitation and transmission curve for filter.

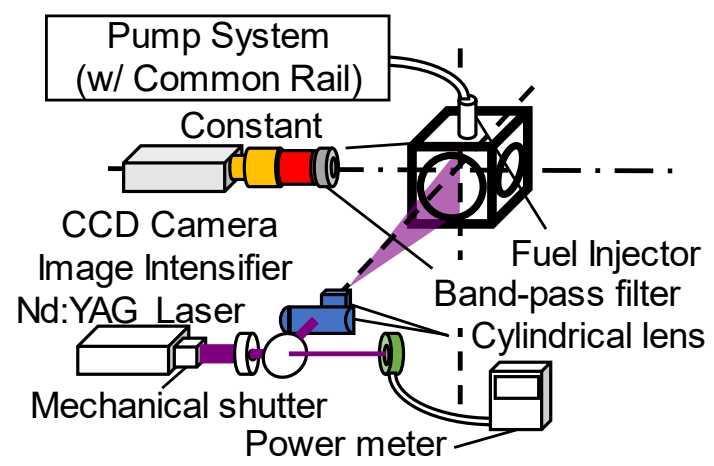

Figure 2. Optical system for simultaneous measurement of fluorescence from the spray using bandpass filters.

Table 1. Experimental Conditions

\begin{tabular}{c|c}
\hline \hline Test Fuel [vol\%] & nC13:93 vol\%, Tetralin:7 vol\% \\
\hline Test nozzle & Single hole nozzle $(1-\Phi 0.133 \mathrm{~mm})$ \\
\hline Nozzle configuration $/ / d[-]$ & 6.5 \\
\hline Injection Pressure $\Delta P_{i n j}[\mathrm{MPa}]$ & $100,120, \mathbf{1 4 0 , 1 6 0}$ \\
\hline Injection Duration $t_{i n j}[\mathrm{~ms}]$ & 5.0 \\
\hline Ambient Gas & $\mathrm{N}_{2}$ \\
\hline Ambient pressure $P_{a}[\mathrm{MPa}]$ & $1.0, \mathbf{1 . 5}, 2.0,2.5$ \\
\hline Ambient density $\rho_{a}\left[\mathrm{~kg} / \mathrm{m}^{3}\right]$ & $11.2, \mathbf{1 6 . 8}, 22.5,28.1$ \\
\hline Ambient Temperature $T_{a}[\mathrm{~K}]$ & 293 \\
\hline
\end{tabular}


pressure $\Delta P_{i n j}$ was set to four conditions based on $140 \mathrm{MPa}$, and the ambient density $\rho_{a}$ was set to four conditions based on $16.8 \mathrm{~kg} / \mathrm{m}^{3}$. Figure 3 shows the relationship between the injection rate measured by Bosch injection rate measurement method and the laser shooting timing.

\section{Quantitative Analysis Method for Liquid-Phase Concentration}

The relationship between the incident light intensity and the fluorescence intensity, ignoring the attenuation of the incident light due to scattering and absorption by the droplet, is generally given by Equation (1), while the quantum yield is given by Equation (2).

$$
\begin{aligned}
& F=K I_{i} V C B \emptyset \\
& \emptyset=\frac{k_{f}}{k_{f}+k_{Q 1}+k_{i s c}}
\end{aligned}
$$

Where $F$ is the fluorescence intensity, $K$ is the optical constant, $l_{i}$ is incident laser light intensity, $\mathrm{V}$ is the volume, $B$ is the transition probability to the excited state, $C$ is liquid phase concentration, $\Phi$ is the quantum yield, $k_{f}$ is the rate of fluorescence emission, $k_{Q 1}$ is the rate of radiation-free transition and intramolecular vibrational conversion, and $k_{\text {isc }}$ is the rate of interterm crossing to the triplet. It has been confirmed in experiments with single droplets that the fluorescence intensity is proportional to the droplet mass, and the relationship is applicable to spray fields as well [5][6]. However, the thickness of the laser sheet light is about $0.4 \mathrm{~mm}$, which is larger than the diameter of the nozzle diameter, and it is said that there is an effect of multiple scattering in the area with high number density up to about $10 \mathrm{~mm}$ from the nozzle diameter. It is also known that the fluorescence intensity is temperature and pressure dependent. In this study, the initial temperatures of the fuel and the ambient fluid are set to be constant, and the effects of evaporation are negligible, so there is no temperature distribution in the field to be measured, and the pressure distribution is considered to be uniform. Therefore, Equation (3), which simplifies Equation (1) and sets $K^{\prime}$ as the proportionality constant between the fluorescence intensity and the liquid phase concentration, is used.

$$
F=K^{\prime} C
$$

In this quantitative analysis, the mass flow rate of the fuel in the cross section perpendicular to the spray axis is used. Therefore, the velocity distribution on the cross section including the central axis of the spray to be measured is necessary. Since the spatial distribution of velocity

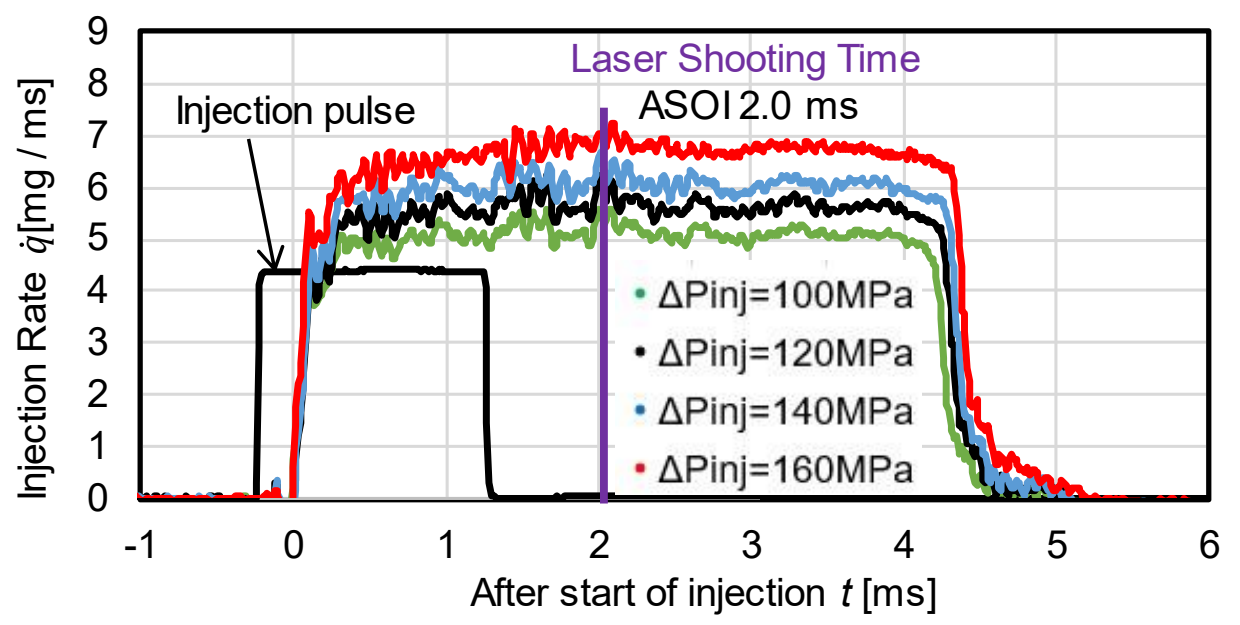

Figure 3. Relation between rate of injection profiles and laser firing time with different injection pressures. The laser shooting time is $2.0 \mathrm{~ms}$ after the start of injection so that the injection rate and the spray in the visible region become quasi-steady state. 
was not measured directly, it was estimated from the relationship between concentration and velocity using momentum theory. The relationship between the test volume and the volumes of fuel and ambient gas is shown in Equation (4). The relationship between the test volume and the volumes of fuel and ambient gas is expressed in Equation (4), and the relationship in Equation (5) is given by momentum theory [7].

$$
\begin{aligned}
& V_{\text {cell }}=V_{a}+V_{f}=\frac{m_{a}}{\rho_{a}}+\frac{m_{f}}{\rho_{f}} \\
& M_{f} v_{0}=\left(M_{f}+M_{a}\right) v
\end{aligned}
$$

where $V_{\text {cell: }}$ test volume $\left[\mathrm{m}^{3}\right], V_{a}$ : volume of ambient gas in the test volume $\left[\mathrm{m}^{3}\right], V_{f}$ : volume of fuel in the test volume $\left[\mathrm{m}^{3}\right], m_{a}$ : mass of ambient gas in the test volume $[\mathrm{kg}], m_{f}$ : mass of fuel in the test volume $[\mathrm{kg}], \rho_{a}$ : ambient density $\left[\mathrm{kg} / \mathrm{m}^{3}\right], \rho_{f}$. fuel density $\left[\mathrm{kg} / \mathrm{m}^{3}\right], M_{f}$ : initial mass of the spray element $[\mathrm{kg}], M_{a}$ : mass of ambient gas entrained in the spray element $[\mathrm{kg}], v_{0}$ : initial injection velocity of the spray element $[\mathrm{m} / \mathrm{s}], v$ : spray axial velocity of the spray element mixture $[\mathrm{m} / \mathrm{s}]$. By taking the air-fuel ratio from Equation (5), the relationship between the concentration $C\left[\mathrm{~kg} / \mathrm{m}^{3}\right]$ and the velocity $v[\mathrm{~m} / \mathrm{s}]$ is given, and from Equations (3) and (4), the relationship in Equation (6) is obtained [8].

$$
\rho_{a}\left(\frac{K^{\prime}}{F}-\frac{1}{\rho_{f}}\right)=\frac{v_{0}}{v}-1
$$

The injection velocity $v_{0}[\mathrm{~m} / \mathrm{s}]$ was obtained from the theoretical injection velocity calculated from the Bernoulli Equation multiplied by the average value of the discharge coefficient measured by the Bosch injection rate measurement method under quasi-steady state conditions. The mass flow rate of the fuel in the cross section perpendicular to the spray axis is calculated from Equation (7) by assuming that the spray is axisymmetric with the spray axis as the central axis. The relationship with the measured fluorescence intensity is given by Equation (8).

$$
\begin{aligned}
& \dot{m}_{f}=\int_{r=0}^{r=\infty} 2 \pi r \cdot C \cdot v d r \\
& \dot{m}_{f}=\int_{r=0}^{r=\infty} 2 \pi r \cdot \frac{F}{K^{\prime}} \cdot \frac{C_{d} \sqrt{\frac{2 \Delta p_{\text {inj }}}{\rho_{f}}}}{1+\rho_{a}\left(\frac{K^{\prime}}{F}-\frac{1}{\rho_{f}}\right)} d r
\end{aligned}
$$

where $\dot{m}_{f}$ : mass flow rate of fuel in the cross section perpendicular to the spray axis $[\mathrm{kg} / \mathrm{s}]$, $C_{d}$ : discharge coefficient [-]. Assuming that the mass flow rate of the fuel in the cross-section perpendicular to the spray axis is the same as the injection rate, the proportionality constant $K^{\prime}$ between the fluorescence intensity and the liquid concentration in each cross-section is obtained by substituting the measured injection rate into the left-hand side of Equation (8). Assuming that the mass flow rate of the fuel in the cross-section perpendicular to the spray axis is the same as the injection rate, the proportionality constant $K^{\prime}$ between the fluorescence intensity and the liquid concentration in each cross-section is obtained by substituting the measured injection rate into the left-hand side of Equation (8). Although $K^{\prime}$ has a distribution in the spray axial distance due to the thickness of the laser sheet and the effect of multiple scattering [2], it is a constant value with fluctuations in the range where the method can be applied. Figure 4 shows the spatial distribution of $K^{\prime}$ with distance from the nozzle tip along the spray axis for different fuel injection pressures, and Figure 5 shows the spatial distribution of $K^{\prime}$ for different ambient densities. The position where the spray spreads sufficiently and is no longer affected by the thickness of the laser sheet light corresponds to the axial distance of about $5 \mathrm{~mm}$ from the nozzle tip. The effect of multiple scattering decreases as the spray axial distance from the nozzle tip increases, and the effect is negligible above about $20 \mathrm{~mm}$. Therefore, the liquid phase concentration distribution was calculated from the fluorescence 
intensity distribution of the captured images using the average value of $K^{\prime}$ from the nozzle tip to the spray axial distance of $20 \mathrm{~mm}$.

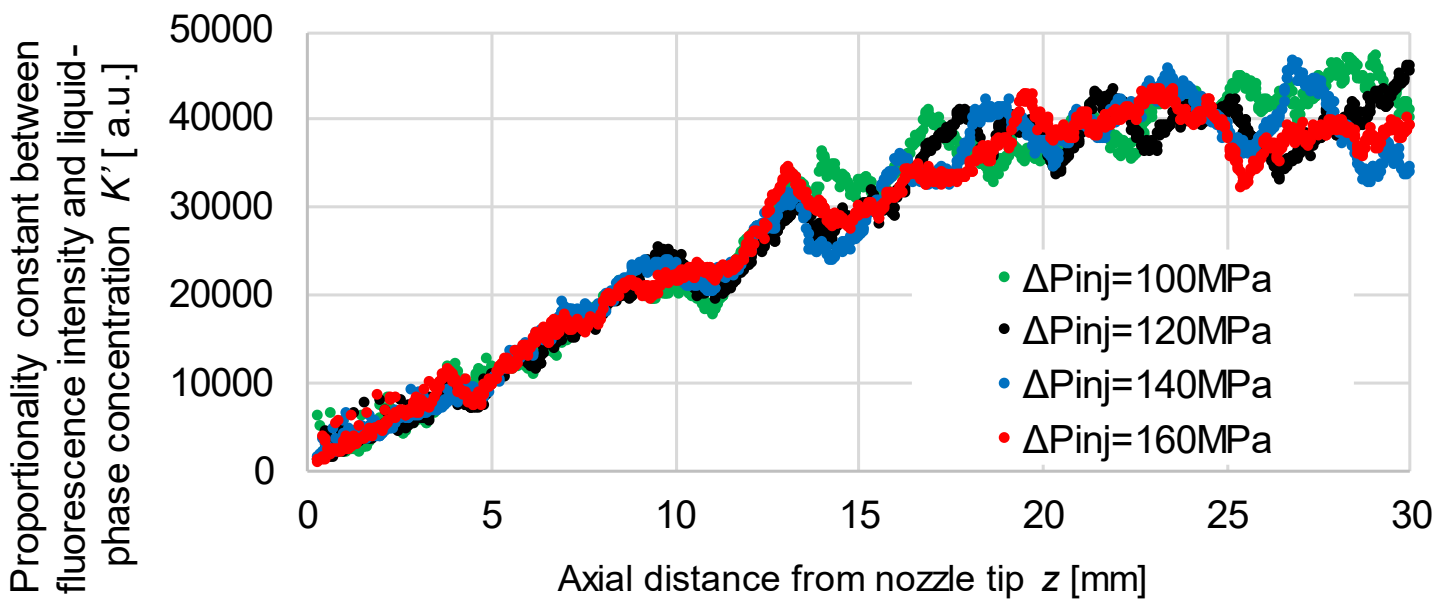

Figure 4. Spatial distribution of $K^{\prime}$ with distance from the nozzle tip along the spray axis for different fuel injection pressures.
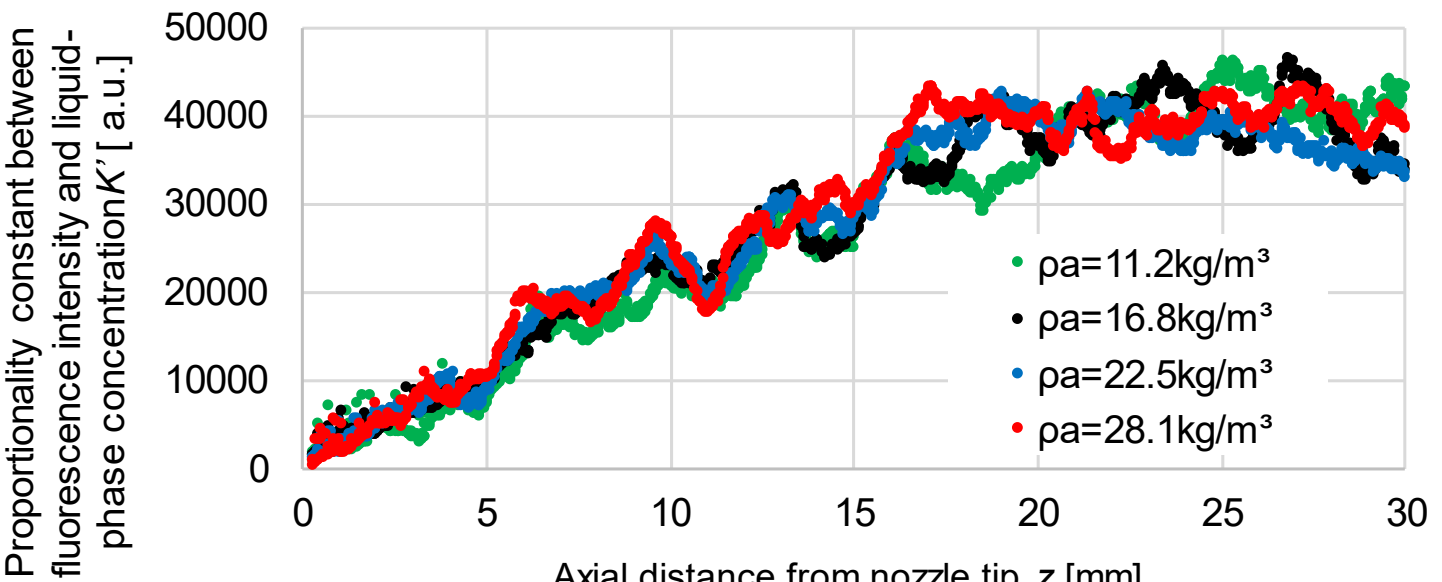

Axial distance from nozzle tip $z[\mathrm{~mm}]$

Figure 5. Spatial distribution of $K^{\prime}$ with distance from the nozzle tip along the spray axis for different ambient densities.

\section{Distribution Characteristics of Liquid-Phase Concentration}

The distribution of liquid-phase concentration at different fuel injection pressures and ambient densities are shown in Figure 6(a) and Figure 6(b), respectively. In addition to the liquidphase concentration, equivalent ratio, which were calculated using air as the ambient gas, are also indicated. In all fuel injection pressures and ambient density conditions, the dense liquid phase in the center of the spray near the nozzle dispersed radially as the distance from the nozzle tip increased, forming a low-density region around the center of the spray. Figure 7 shows the radial equivalent ratio distribution in the cross section perpendicular to the spray axis at the position where the distance from the nozzle is 200 times the hole diameter $(z=26.6$ $\mathrm{mm}$ ) with changing the fuel injection pressure. Increasing the fuel injection pressure had little effect on the spray spread and concentration distribution. Although increasing the fuel injection pressure increased the injection rate, the liquid phase concentration remained relatively similar due to the increase in the amount of air entrainment. Figure 8 shows the radial equivalent ratio distribution in the cross section perpendicular to the spray axis at the position 
where the distance from the nozzle is 200 times the hole diameter ( $z=26.6 \mathrm{~mm}$ ) with changing the ambient densities. The spray image shows that the spray spreads as the ambient density increases. In addition, as the ambient density increases, the equivalence ratio decreases at the same concentration, resulting in a large decrease in the equivalence ratio in the spray.

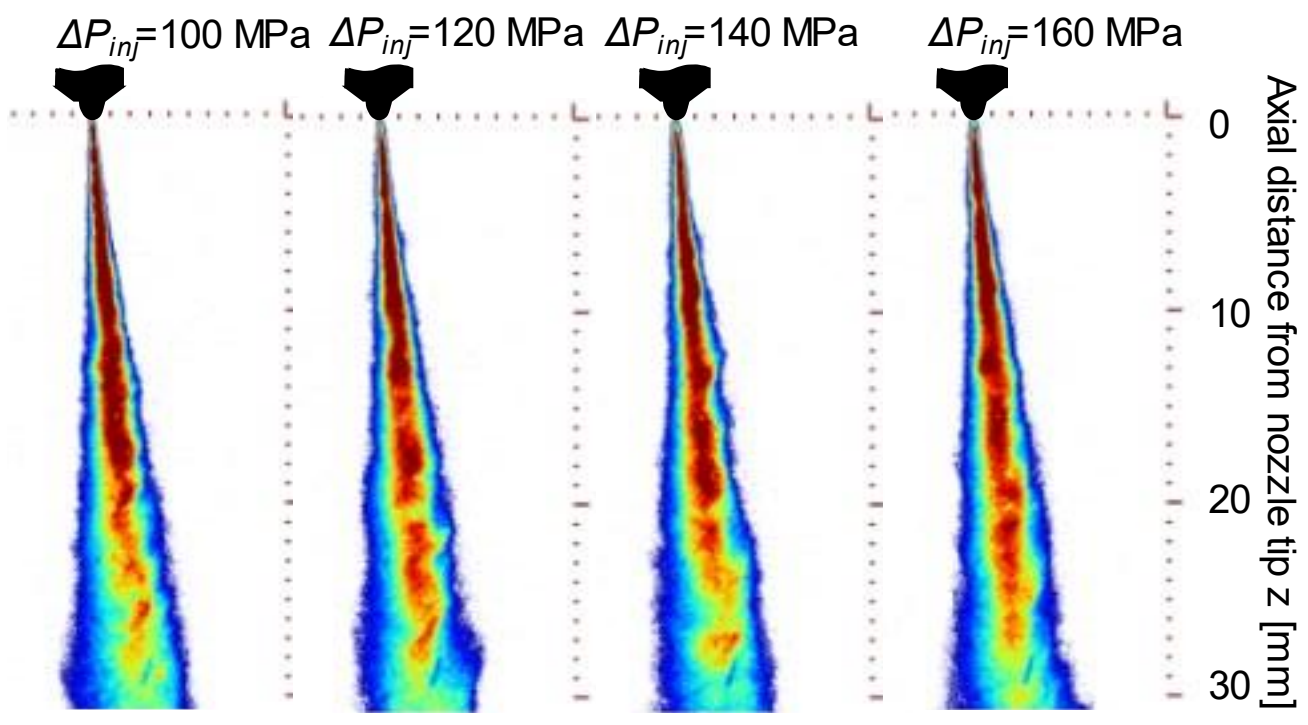

(a) Changing fuel injection pressure $\left(\rho_{a}=16.8 \mathrm{~kg} / \mathrm{m}^{3}\right)$

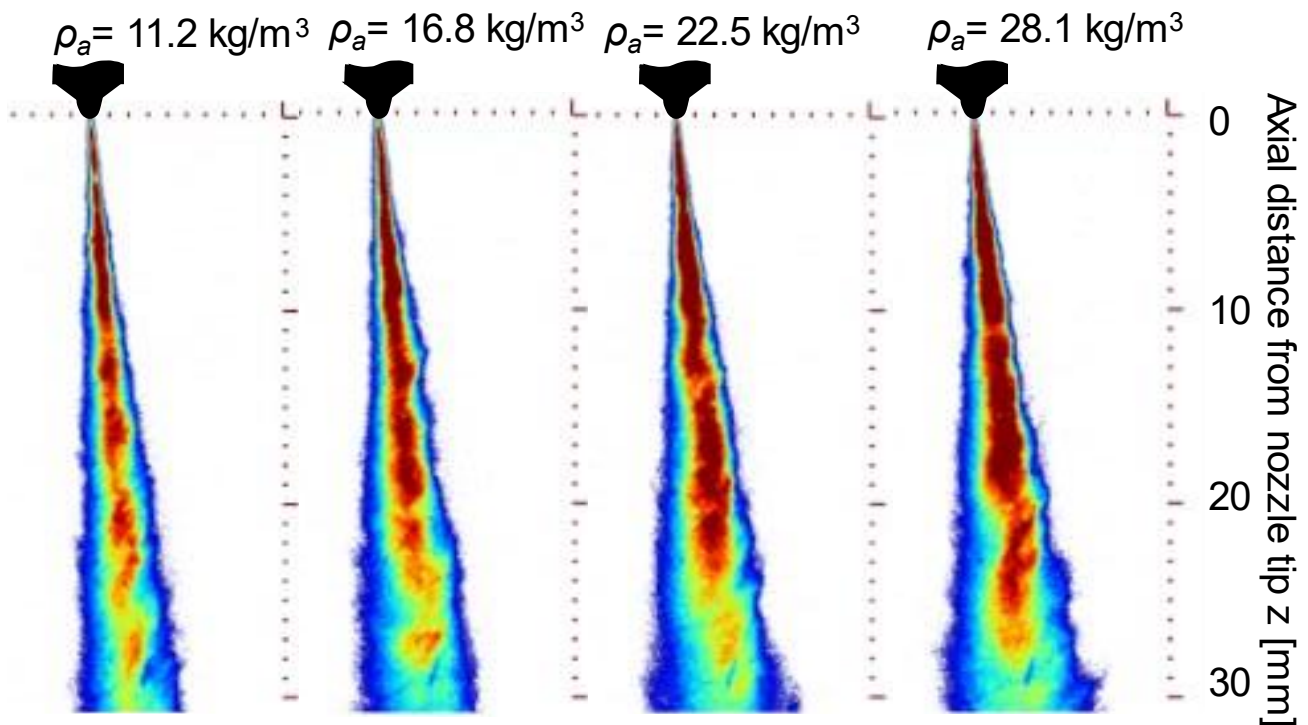

(b) Changing ambient density $\left(\Delta P_{i n}=140 \mathrm{MPa}\right)$

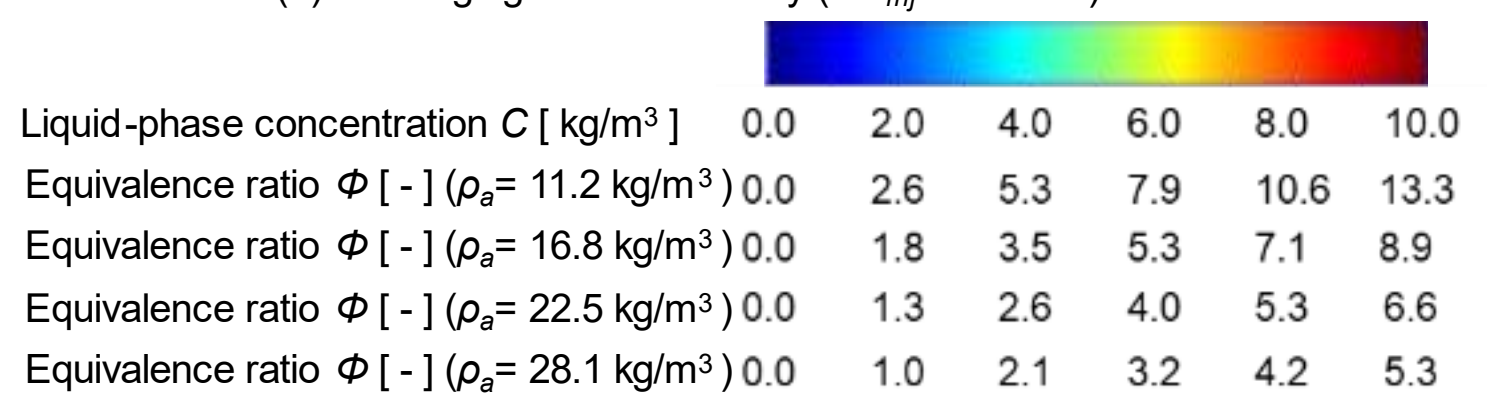

Figure 6. Distribution of liquid-phase concentration at different fuel injection pressures and ambient densities after start of injection $2.0 \mathrm{~ms}$. (a) Changing $\Delta P_{i n j}\left(\rho_{a}=16.8 \mathrm{~kg} / \mathrm{m}^{3}\right)$, (b) Changing $\rho_{a}\left(\Delta P_{i n j}\right.$ $=140 \mathrm{MPa})\left(\Phi d=0.133 \mathrm{~mm}, T_{a}=293 \mathrm{~K}\right)$ 


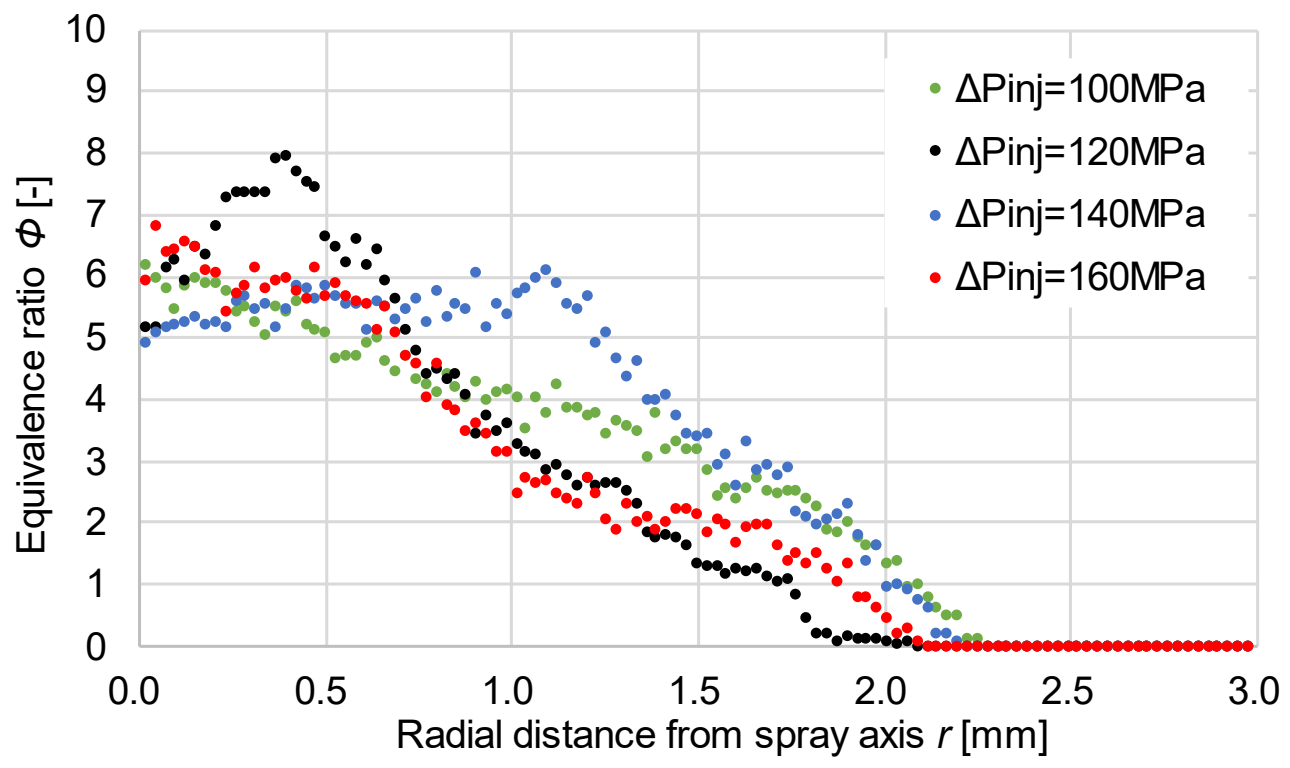

Figure 7. Radial equivalent ratio distribution in the cross section perpendicular to the spray axis at the position where the distance from the nozzle is 200 times the hole diameter $(z=26.6 \mathrm{~mm})$ with changing the fuel injection pressure. $\left(\rho_{a}=16.8 \mathrm{~kg} / \mathrm{m}^{3}, \Phi d=0.133 \mathrm{~mm}, T_{a}=293 \mathrm{~K}\right)$

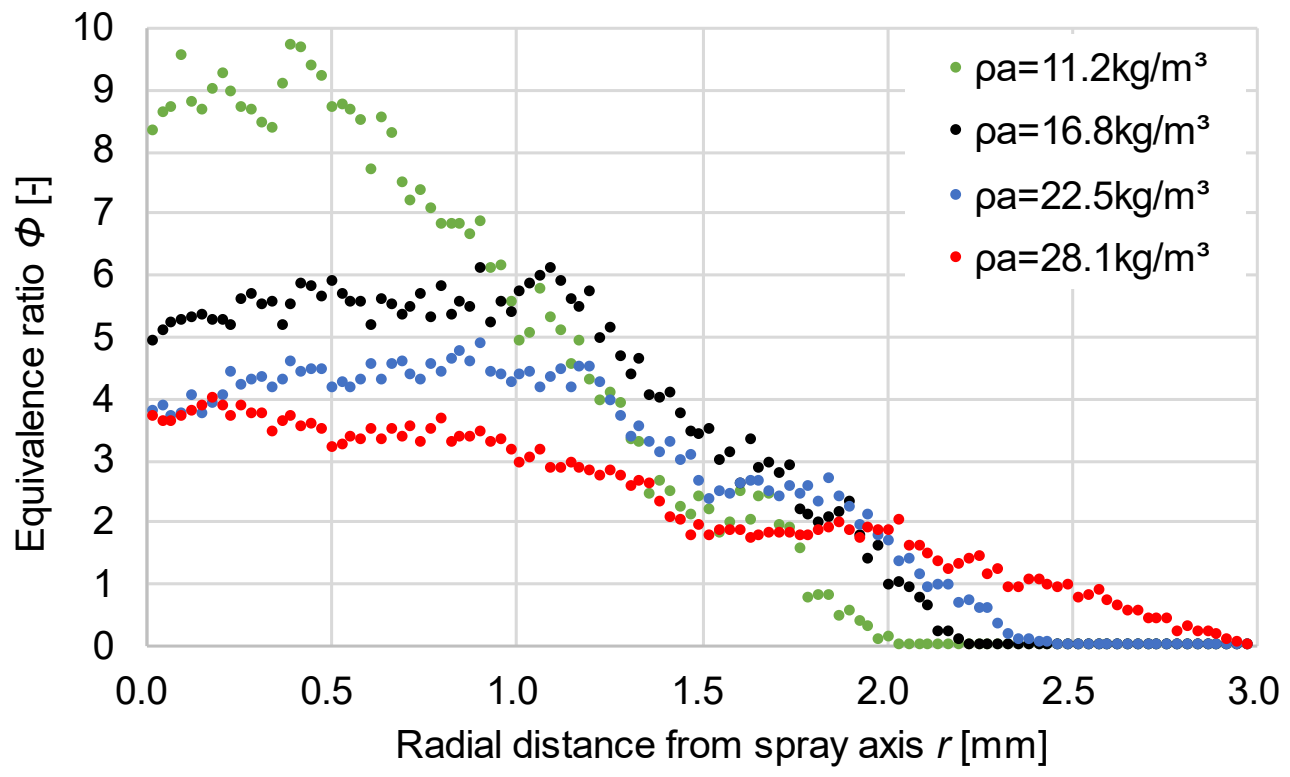

Figure 8. Radial equivalent ratio distribution in the cross section perpendicular to the spray axis at the position where the distance from the nozzle is 200 times the hole diameter $(z=26.6 \mathrm{~mm})$ with changing the ambient densities. $\left(\triangle P_{i n j}=140 \mathrm{MPa}, \Phi d=0.133 \mathrm{~mm}, T_{a}=293 \mathrm{~K}\right)$

\section{Conclusions}

In this paper, a quantitative analysis method of liquid-phase concentration distribution is proposed by applying the laser-induced fluorescence method to a non-evaporating diesel spray. The method was used to analyse the spray under different fuel injection pressures and ambient densities. As a result, it was found that the effect of the fuel injection pressure on the concentration distribution was small, but the spray spreads and the equivalent ratio became decrease with the increase of the ambient density. 


\section{Nomenclature}

C Liquid phase concentration $\left[\mathrm{mol} / \mathrm{m}^{3}\right]$

$c_{a} \quad$ Specific heat of the ambient gas $[\mathrm{J} /(\mathrm{kg} \cdot \mathrm{K})]$

$C_{f v} \quad$ Specific heat of the gaseous fuel $[\mathrm{J} /(\mathrm{kg} \cdot \mathrm{K})]$

$C_{f l} \quad$ Specific heat of liquid fuel $[\mathrm{J} /(\mathrm{kg} \cdot \mathrm{K})]$

$F \quad$ Fluorescence intensity [a.u.]

$K \quad$ Optical constant [a.u.]

$K^{\prime} \quad$ proportionality constant [a.u.]

$l_{i} \quad$ Incident laser light intensity [mJ]

$M_{a} \quad$ Mass of ambient gas entrained in the spray element [kg]

$M_{f} \quad$ Initial mass of the spray element [kg]

$\dot{m}_{f} \quad$ Mass flow rate of fuel in the cross section perpendicular to the spray axis [kg/s]

$I_{i} \quad$ Incident laser light intensity [mJ]

$\dot{q} \quad$ Injection rate $[\mathrm{mg} / \mathrm{ms}]$

$v_{0} \quad$ Initial injection velocity of the spray element $[\mathrm{m} / \mathrm{s}]$

$v \quad$ Spray axial velocity of the spray element mixture $[\mathrm{m} / \mathrm{s}]$

$V_{a} \quad$ Volume of ambient gas in the test volume $\left[\mathrm{m}^{3}\right]$

$V_{\text {cell }} \quad$ Test volume $\left[\mathrm{m}^{3}\right]$

$V_{f} \quad$ Volume of fuel in the test volume $\left[\mathrm{m}^{3}\right]$

$l_{i} \quad$ Volume of ambient gas in the test volume $\left[\mathrm{m}^{3}\right]$

$\rho_{a} \quad$ Density of the ambient gas $\left[\mathrm{kg} / \mathrm{m}^{3}\right]$

$\rho_{f} \quad$ Density of the fuel $\left[\mathrm{kg} / \mathrm{m}^{3}\right]$

$\tau \quad$ Transmittance [-]

\section{References}

[1] Yokota, H., Kamimoto, T., Kosaka, H., and Tsujimura, K., 1991, SAE Technical Paper 910225.

[2] Yamane, Y., Yokota, H. and Kamimoto, T.,1992, Transactions of the Japan Society of Mechanical Engineers Series B, Vol.58, No.550, pp.1955-1960.

[3] Yamane, K., Ikegami, M., and Shioji, M.,1994-7, Proc. of the 3rd International Symposium on Diagnostics and Modeling of Combustions in Internal Combustion Engines (COMODIA94), 225-230

[4] Kawano, D., Senda, J., Kawakami, K., Shimada, A., and Fujimoto, H.,2002, SAE Technical Paper 2002-01-0220.

[5] Domann, R. and Hardalupas, Y., Part. Part. Syst. Charact., 2001, 18, pp.3-11.

[6] Melton, L.A., U.S.A.O., Contract DAAL03-86-K-0082, Univ. Texas at Dallas, $1988,5$.

[7] Wakuri, Y., Fujii, M., Amiyani, T. and Tsuneya, R.,1959, Transactions of the Japan Society of Mechanical Engineers, Vol.25, No.156, pp.820-826.

[8] Zama, Y., Ochiai, W., Sugawara, K., Furuhata, T. and Arai, M., 2013, Atomization and Sprays. 23, pp.443-461. 